\title{
Driving Fatigue Recognition with Functional Connectivity Based on Phase Synchronization
}

\author{
Hongtao Wang, Member, IEEE, Xucheng Liu, Junhua Li, Senior Member, IEEE, Tao Xu, \\ Anastasios Bezerianos, Senior Member, IEEE, Yu Sun*, Senior Member, IEEE and Feng Wan*, Senior \\ Member, IEEE
}

\begin{abstract}
Accumulating evidences showed that the optimal brain network topology was altered with the progression of fatigue during car driving. However, the extent of discriminative power of functional connectivity that contribute to the driving fatigue detection is still unclear. In this study, we extracted two types of features (network properties and critical connections) to explore their usefulness in driving fatigue detection. EEG data were recorded twice from twenty healthy subjects during a simulated driving experiment. Multi-band functional connectivity matrices were established using phase lag index, which serve as input for the following graph theoretical analysis and critical connections determination between the most vigilant and fatigued states. We found a reorganisation of brain network towards less efficient architecture in fatigue state across all frequency bands. Further interrogations showed that the discriminative connections were mainly connected to frontal areas, i.e., most of the increased connections are from frontal pole to parietal or occipital regions. Moreover, we achieved a satisfactory classification accuracy $(96.76 \%)$ using the discriminative connection features in $\beta$ band. Our study demonstrated that graph theoretical properties and critical connections are of discriminative power for manifesting fatigue alterations and the critical connection is an efficient feature for driving fatigue detection.
\end{abstract}

Index Terms-Driving Fatigue, Electroencephalogram, Func-

This study was supported by the Natural Science Foundation of Guangdong Province (Grant no. 2018A030313882), Projects for International Scientific and Technological Cooperation (Grant no. 2018A05056084), Jiangmen Brainlike Computation and Hybrid Intelligence R\&D Center ([2018]359, [2019]26), National Natural Science Foundation of China (Grant no. 61806149), and the Science Foundation for Young Teachers of Wuyi University (Grant no. 2018td01).Yu Sun was supported by the National Natural Science Foundation of China (81801785), the Fundamental Research Funds for the Central Universities (2018QNA5017, 2019FZJD005), and the Hundred Talents Program of Zhejiang University. This work was partially supported by the grants from the Zhejiang Lab (no. 2019KE0AD01), the Science and Technology Development Fund, Macau SAR (File no. 055/2015/A2, 0045/2019/AFJ) and the University of Macau Research Committee (MYRG: 2016-00240-FST, 2017-00207-FST). (Hongtao Wang and Xucheng Liu contributed equally to this work.), (*, Corresponding author: Yu Sun, Feng Wan)

H. Wang, X. Liu and X. Tao are with the Faculty of Intelligent Manufacturing, Wuyi University, Jiangmen, 529020, China.

J. Li is with School of Computer Science and Electronic Engineering, University of Essex, Colchester, United Kingdom, also with the Faculty of Intelligent Manufacturing, Wuyi University, Jiangmen 529020, China.

A. Bezerianos is with the N1 Institute, National University of Singapore, 117456, Singapore and also with the Dept of Medical Physics, University of Patras Greece, 26500 Patras, Greece.

Y. Sun is with the Key Laboratory for Biomedical Engineering of Ministry of Education of China, Department of Biomedical Engineering, Zhejiang University, and with the Department of Radiology, Children's Hospital, Zhejiang University School of Medicine, National Clinical Research Center for Child Health, 310052, Hangzhu, China, (email: yusun@zju.edu.cn).

F. Wan is with the Department of Electrical and Computer Engineering, Faculty of Science and Technology, University of Macau, Macau, and also with the Centre for Cognitive and Brain Sciences, Institute of Collaborative Innovation, University of Macau, Macau (e-mail:fwan@um.edu.mo). tional Connectivity, Graph Theory

\section{INTRODUCTION}

D RIVERS, especially commercial truck drivers who transport cargo over long distance, sometimes have to drive for a prolonged time. The prolonged driving could lead to mental fatigue and vigilance decline, which might result in faults of driving operations [1], even traffic accidents. Distinct from physical fatigue caused by overwork of muscular or cardiovascular system, the mental fatigue is associated with overload of the brain, such as the prolonged implementation of a sustained cognitive task or vigilance task [2]. Drivers might do wrong operations with the state of alert, while under the fatigue state the probability of doing wrong operations is dramatically increased. It has been pointed out that driving fatigue is one of the leading causes for fatal traffic accidents and is considered to account for $20-30 \%$ of all the vehicle accidents [3]. Therefore, it is necessary to develop efficient approaches for detecting driving fatigue with a high accuracy(e.g., [4]), reducing the traffic accidents caused by driving fatigue.

Compared to the real driving, a simulated driving in a laboratory provides a more feasible way to study drivingrelated mental fatigue as it is under control and secure to participants [5], [6]. Similar to actual driving, the simulated driving is also able to induce driving fatigue by a prolonged cognitive-demand task [7]. Previous studies used eye blinking frequency [8], [9], facial expression [10], or driving performance such as lane keeping and distance keeping [11] to detect driving fatigue. Unfortunately, these methods could be seriously affected by ambient factors and drivers false fatigue behaviors.

In the past decades, techniques monitoring driving fatigue based on physiological signals such as EEG, ECG, and EOG, were developed [12]-[14]. Among these physiological signals, EEG signal which directly reflects the inherent neural activities originated from the brain, has been proven to be one of the most predictive and reliable indicators [15], [16]. EEG signal contains abundant information of human cognitive states. There have been a number of studies which utilized EEG signal to detect drowsiness or mental fatigue [17]-[19]. Roughly, there are four categories according to different utilizations of the characteristics of the EEG signal, including the power spectrum based analysis [20], [21], the entropy based analysis [22], [23], the brain networks based analysis [24][26], and their combinations [18]. The driving fatigue effects 
are vividly reflected by the spectral features [21]. Specifically, the $\theta$ and $\alpha$ rhythms increases during the alteration from alert to fatigue, while the $\beta$ rhythm decreases [24]. However, power spectrum and entropy cannot reveal the interactions among brain regions and their dynamic properties relevant to the change of driving fatigue.

Currently, the emerging neuroimaging techniques have suggested that the human brain can be represented as an interconnected semi-regular network across multiple brain regions and its function relies on regional dynamic interactions [27], [28]. Functional connectivity is a property of regional information transactions that can be measured by statistical dependencies, such as correlations, coherence, or transfer entropy. By computing the coupling strength, the functional network is constructed to unmask the dynamics upon the structural connectivity and internal properties of the human brain [29]. As an analytical approach to explore functional connectivity, graph theory has been utilized to have informative findings about mental state, such as working memory [30], sustained attention tasks [31], [32], and visual attention tasks [33]. Evidence from these studies supports that the human brain possesses a specific topological structure (e.g., small world architecture [34]). Graph theory enables the assessment of functional connectivity in terms of local segregation and global integration [27]. Furthermore, specific brain regions play the essential role in the mental fatigue task. Lim et al. [35] investigated the psychomotor vigilance test $(P V T)$ based on arterial spin labeling (ASL) fMRI data suggesting frontoparietal regions were impacted by demanding of vigilance and continuous attention performance. Liu et al. [36] employed directed transfer function (DTF) to estimate the EEG based functional connectivity, and suggested that long period cognitive task was associated with the pairwise fronto-parietal coupling, especially in $\theta$ and $\beta$ bands. In the simulated driving task, participants need sustained visual stimulation, thus the occipital region for visual function is necessary [37].

Our previous studies focus on the functional connectivity altercation at a single band, including the lower $\alpha$ (8 $10 \mathrm{~Hz})$ or $\theta(4-7 \mathrm{~Hz})$ band respectively, that employed the EEG based functional connectivity analysis and revealed the reorganization of the brain network while mental fatigue emerged [38], [39]. Especially, the characteristic path length presented a positive correlation with the task duration and the high accuracy $92 \%$ for driving fatigue detection was achieved with the critical connection features. Also, our current review showed that mental fatigue was associated with brain disconnectivity, which was in line with the conception of human connectome [40]. These studies cannot reflect the mental mechanism under different frequency bands. Also, to our knowledge, the studies about driving fatigue detection from other researchers have not focus on the both graph theoretical properties and critical connections to investigate their features in multi-band. Therefore, our research creatively analyzed the mechanism of both characteristics in multi-frequency band. To date, few studies have achieved the efficient practical driving fatigue detection because of the technical barriers such as lacking controllable high density EEG data recording device and reliable feature extraction approach. In this paper, the wireless dry EEG recording device was applied to generate the raw data, and the driving fatigue detection approach based on the network graph theoretical properties and critical connection features, was extended to the multi-frequency (e.g. $\theta, \alpha$, and $\beta$ ) bands .

Comparing functional connectivity properties under alert and fatigue states is a valuable approach for driving fatigue detection. The characterization of human neural activity during mental fatigue remains a big challenge, and the complex nature of fatigue-related neural mechanism needs to be studied further. This paper introduces a novel driving fatigue detection method based on the functional connectivity. The current work aimed to achieve efficient driving fatigue detection during driving a vehicle. We achieved the classification of the connection features and their graph theoretical properties, respectively. The findings of this work may provide novel method for efficient driving fatigue detection, and promote technologies to avoid vehicle accidents.

\section{Method And Materials}

\section{A. Subjects}

Subjects participated in this study were twenty healthy students $($ male/female $=15 / 5$, age $=22.2 \pm 3.2$ years) recruited by an internal advertisement within the National University of Singapore, who had never participated in similar driving fatigue experiments previously. Subjects who had been diagnosed with mental illness or sleep disorders were excluded. All of them are right-handed and reported normal or corrected to normal vision. In order to induce driving fatigue more efficiently, all the experiments were carried out in the afternoon from $1 \mathrm{pm}$ to $5 \mathrm{pm}$ at the Cognitive Engineering Laboratory of Singapore Institute for Neurotechnology (SINAPSE). Subjects were prohibited from consuming caffeine and alcohol approximately 4 and 24 hours respectively before the experiment. All subjects were given the information of the experiment and then signed an informed consent form to agree to their participation. This study was approved by Institutional Review Board of the National University of Singapore.

\section{B. Experimental Scenario}

The simulated driving environment mainly consists of two segments: a simulated driving system and a wireless dry EEG acquisition system, as shown in Fig.1 (a). The driving rules were set up as Singapore traffic regulation to utilize an experimental vehicle, pedals, and simulated driving product (Logitech G27 Racing Wheel, Switzerland). Three 65-inch LCD screens were employed to show a panorama of road condition. The duration of each experiment was one and a half hours with a silent driving condition. The traffic condition includes a perfect straight two-way lane in the rural and minimum vehicle density to make participants feel boring and less challenging. Participants were asked to drive as stable as possible.

A guiding car was in front of the car controlled by participants and suddenly reduced the speed at some random time points during the experiment. The participants had to break the controlled car to avoid collision when the speed of the 
guiding car was reduced. The reaction time (RT), which was the time lapse from the onset of speed reduction of the guiding car to the time point of pressing the break by participants, was recorded.

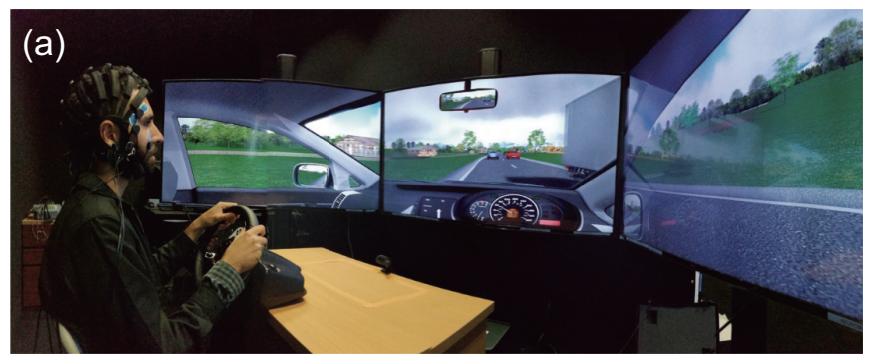

(b)

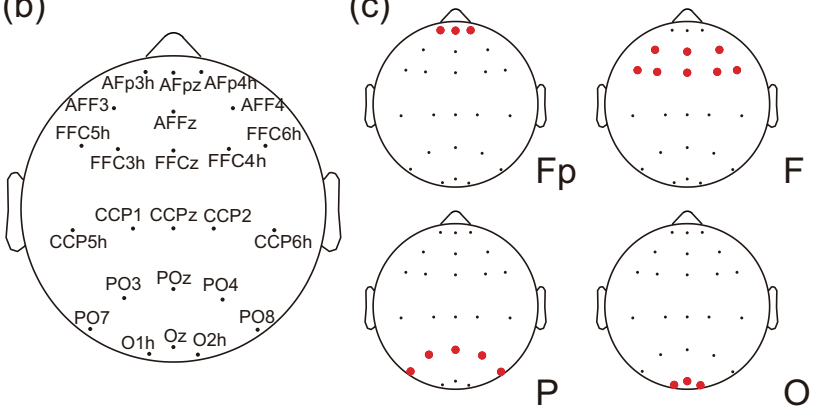

Fig. 1. Experiment setup for simulated driving experiment scenario. (a) is the experimental environment. (b) is the channel location of electrodes. Furthermore, (c) is the subdivision of the EEG channels according to their locations, which would be used for the following determination of driving fatigue-related critical connections. Fp: Frontal pole region; F: Frontal region; P: Parietal region; O: Occipital region.

\section{Data Acquisition and Pre-processing}

During the experiment, each subject was requested to wear a wireless dry EEG headset (model: HD-72, Cognionics Inc., USA) with 24 electrodes, and participated the same driving task twice. Considering the total 20 subjects, 40 sections of the EEG data were included in this paper. As shown in Fig.1 (b), twenty-four dry electrodes were on the headset and referenced to the average of the left and right mastoids. All electrodes were placed according to the international 10-20 system standards [41] and the impedance was kept below 20 $\mathrm{k} \Omega$ during EEG acquisition. EEG signals were digitized at a rate of $250 \mathrm{~Hz}$ with the Cognionics acquisition software. Bipolar electrooculogram (EOG) data were acquired from the outer canthi (Horizontal EOG, HEOG) and the regions above and below the left eye (Vertical EOG, VEOG).

The recorded raw signal was band-pass $(1 \sim 40 \mathrm{~Hz})$ filtered and re-referenced by common average reference (CAR). Due to contaminations by artifacts from eye blinks or muscular activities, the EEG signal has a low signal-to-noise ratio (SNR). To improve it, independent component analysis (ICA) was employed to remove the components representing artifacts [42]. The artifacts-reduced EEG signal was decomposed into frequency bands of $\theta, \alpha$, and $\beta$ by the Discrete Wavelet Transform (DWT) [43]. The Daubechies wavelet of $\mathrm{db} 4$ and decomposition level 6 were employed. The EEG data were decomposed to $2^{j}$ set of independent orthogonal sub-bands with $N$ layer wavelet-packet decomposition. Wavelet coefficient vectors can be computed by:

$$
\left[\frac{(m-1) F_{s}}{2^{j+1}}, \frac{m F_{s}}{2^{j+1}}\right]
$$

where $F_{s}$ is the sampling frequency, in this study $F_{s}=250$ $\mathrm{Hz}$ and $m=0,1,2, \ldots, 2^{j-1}$. According to the sampling frequency $(250 \mathrm{~Hz})$ and computed frequency from 0 to Nyquist frequency $(125 \mathrm{~Hz})$ in the 6th layer, the frequency components of the $\theta, \alpha$, and $\beta$ bands were exacted by restructuring the wavelet-packet signals.

According to the study on behavior performance, the first and last 5 minutes were defined as the alert and fatigue states (detailed are placed in result section). Hence, EEG data within these two blocks were chosen for the following procedures. EEG data were preprocessed by EEGLAB [44] with the inhouse MATLAB script.

\section{Functional Connectivity Network Construction}

After EEG data were efficiently processed according to their oscillatory components into standard $\theta, \alpha$, and $\beta$ bands, the statistical coupling was computed by the method of phase synchronization (PS) family to construct functional connectivity in each frequency band. Consistent with the features of EEG data, the PS was computed the phase synchronization between two time series, even if their amplitudes are independent [45]. We utilized the phase lag index (PLI) to estimate the degree of pairwise coupling for further research. For each band, the EEG signals were divided by dividing the floating time window over the step: window/step $=4 / 2 \mathrm{~s}$.

Considering pair time series $s_{1}(t)$ and $s_{2}(t)$, we computed the instantaneous phase $z_{i}(t)$ with Hilbert transform [46]:

$$
z_{i}(t)=s_{i}(t)+j H T\left(s_{i}(t)\right)
$$

where $H T\left(s_{i}(t)\right)$ is the Hilbert transform of each time series $s_{i}(t)$, which is estimated by:

$$
H T\left(s_{i}(t)\right)=\frac{1}{\pi} P . V . \int_{-\infty}^{\infty} \frac{s_{i}(t)}{t-\tau} d \tau
$$

In Eq.3, P.V. represents Cauchy principal value. Once the phase of each time series is computed, the relative phase locking can be estimated as :

$$
\Delta \varphi(t)=\arg \left(\frac{z_{1}(t) z_{2}^{*}(t)}{\left|z_{1}(t)\right|\left|z_{2}(t)\right|}\right)
$$

The PLI value ranged between 0 and 1 is calculated with following equation:

$$
P L I=|\langle\operatorname{sign} \varphi(t)\rangle|
$$

The PLI value is defined $[0,1]$ with 0 represents the case where there is no phase synchronization, while 1 represents the perfect phase locking between two time series.

\section{E. Graph Theoretical Analysis}

After building the functional connectivity network, $N * N$ adjacency matrices ( $N=24$ in this study) were computed, 
which represent the connectivity structures of brain nodes. Since the functional connectivity network contains complex information and numerous useless combinations, the sparsity ranged from $10 \%$ to $20 \%$ with the step of $1 \%$ was utilized to these networks, which is the ratio of present connection number to preserve as the real functional connections. Thus, the weighted adjacency matrices were computed, which preserved the connection strength of the real connections. For the graph theoretical properties with the considered sparsity, the area under curve of the corresponding properties were extracted as features for further driving fatigue detection.

In order to quantitatively investigate the topological properties of functional connectivity between different mental states (alert and fatigue), we implemented graph theoretical analysis with the Brain Connectivity Toolbox [47]. We characterized the graph in the aspects of local segregation (clustering coefficient $\left(C^{w}\right)$ and local efficiency $\left.\left(E_{\text {local }}^{w}\right)\right)$ and global integration (characteristic path length $\left(L^{w}\right)$ and global efficiency $\left(E_{\text {global }}^{w}\right)$ and small world-ness $(S W)$ based on the weighted adjacency matrices.

For the weighted and undirected adjacency matrices $G$ with $N$ nodes, each electrode was defined as the node of the graph in this study. $C^{w}$ is the main graph index of information segregation in networks, which is calculated as [48]:

$$
C^{w}=\frac{1}{n} \sum_{i \in N} \frac{2 t_{i}^{w}}{k_{i}\left(k_{i}-1\right)}
$$

where $t_{i}^{w}$ is the number of links, which is defined weighted geometric mean of triangles around node $i$ between the neighbors, and $k_{i}$ is the number of connected nodes of $i$. $C^{w}$ reflects the tendency of a network to form topologically local circuits and the higher value of $C^{w}$ represents the more connections of node $i . L^{w}$ is the mean of shortest path length, which is the path with the largest total weight between the vertices, defined as:

$$
L^{w}=\frac{1}{n} \sum_{i \in N} \frac{\sum_{j \in N, j \neq i} d_{i j}^{w}}{n-1}
$$

In Eq.7, $d_{i j}^{w}$ is the shortest weighted path length between $i$ and $j . L^{w}$ is the main measure for the global integration of the graph. SW is defined as:

$$
S W=\frac{C^{w} / C_{\text {rand }}^{w}}{L^{w} / L_{\text {rand }}^{w}}
$$

where $L_{\text {rand }}^{w}$ and $C_{\text {rand }}^{w}$ were calculated by the mean of $C^{w}$ and $L^{w}$ of 100 random surrogate random networks, which had the equal degree, nodes and edges with the subject's functional network. The random networks were reconstructed by rebuilding the edges in the graph [49]. SW measures the balance between local segregation and global integration [50].

$E_{\text {global }}^{w}$ measures the overall information exchange capability of the network, which is defined as the average inverse shortest path length:

$$
E_{\text {global }}^{w}=\frac{1}{n} \sum_{i \in N} \frac{\sum_{j \in N, j \neq i}\left(d_{i j}^{w}\right)^{-1}}{n-1}
$$

$E_{\text {local }}^{w}$ is a measurement to estimate the efficiency of information transactions within the regional component in a network. $E_{\text {local }}^{w}$ is defined as:

$$
E_{\text {local }}^{w}=\frac{1}{2} \sum_{i \in N} \frac{\sum_{j, h \in N, j \neq i}\left(w_{i j} w_{i h}\left[d_{j h}^{w}\left(N_{i}\right)\right]^{-1}\right)^{1 / 3}}{k_{i}\left(k_{i}-1\right)}
$$

where $w_{i j}$ is connection weights between node $i$ and $j$.

TABLE I

THE GRAPH THEORETICAL PROPERTIES IN DIFFERENT MENTAL STATES.

\begin{tabular}{ccccccc}
\hline \multirow{2}{*}{ Frequency band } & Method & Alert (S.D.) & Fatigue (S.D.) & \multicolumn{3}{c}{ Statistical analysis } \\
\cline { 5 - 7 } & & & $F_{1,78}$ & $p-$ Value \\
\hline \multirow{3}{*}{ Theta } & $C^{w}$ & $0.019(0.003)$ & $0.020(0.004)$ & 3.727 & 0.057 & 0.046 \\
& $L^{w}$ & $0.648(0.091)$ & $0.691(0.072)$ & 5.621 & $\mathbf{0 . 0 2 0}$ & 0.067 \\
& $E_{\text {global }}^{w}$ & $0.023(0.001)$ & $0.022(0.002)$ & 5.776 & $\mathbf{0 . 0 1 9}$ & 0.069 \\
& $E_{\text {local }}^{w}$ & $0.016(0.004)$ & $0.018(0.005)$ & 5.499 & $\mathbf{0 . 0 2 2}$ & 0.066 \\
& $S W$ & $0.193(0.031)$ & $0.203(0.034)$ & 1.919 & 0.170 & 0.024 \\
\hline \multirow{3}{*}{ Alpha } & $C^{w}$ & $0.018(0.003)$ & $0.020(0.003)$ & 4.173 & $\mathbf{0 . 0 4 4}$ & 0.051 \\
& $L^{w}$ & $0.685(0.096)$ & $0.736(0.078)$ & 6.734 & $\mathbf{0 . 0 1 1}$ & 0.079 \\
& $E_{\text {global }}^{w}$ & $0.023(0.001)$ & $0.022(0.002)$ & 5.723 & $\mathbf{0 . 0 1 9}$ & 0.068 \\
& $E_{\text {local }}^{w}$ & $0.015(0.004)$ & $0.022(0.002)$ & 6.749 & $\mathbf{0 . 0 1 1}$ & 0.080 \\
& $S W$ & $0.196(0.032)$ & $0.206(0.035)$ & 1.692 & 0.697 & 0.021 \\
\hline \multirow{3}{*}{ Beta } & $C^{w}$ & $0.017(0.003)$ & $0.019(0.004)$ & 4.414 & $\mathbf{0 . 0 3 9}$ & 0.054 \\
& $L^{w}$ & $0.737(0.116)$ & $0.811(0.096)$ & 9.532 & $\mathbf{0 . 0 0 3}$ & 0.109 \\
& $E_{\text {global }}^{w}$ & $0.022(0.001)$ & $0.022(0.002)$ & 4.449 & $\mathbf{0 . 0 3 8}$ & 0.054 \\
& $E_{\text {local }}^{w}$ & $0.014(0.004)$ & $0.016(0.004)$ & 6.868 & $\mathbf{0 . 0 1 1}$ & 0.081 \\
\hline
\end{tabular}

Data were analyzed using one-way ANOVA, and the values are mean with standard deviation (S.D.). $C^{w}$ represents the clustering coefficient. $L^{w}$ represents the characteristic path length. $E_{\text {global }}^{w}$ represents the global efficiency. $E_{\text {local }}^{w}$ represents the local efficiency. $S W$ represents the small worldness. These graph theoretical properties were the area under curve with the sparsity ranged from $10 \%$ to $20 \%$. Significant differences $(p<0.05)$ between alert and fatigue state are highlighted in bold. 


\section{F. Mental Fatigue Classification}

We performed a classification of different mental states (alert and fatigue) basing on the subject-independent discriminative connection features (the PLI value) and the graph theory properties $\left(C^{w}, L^{w}, E_{\text {global }}^{w}, E_{\text {local }}^{w}, S W\right)$. Subjectindependent discriminative connections were selected by using the sequential floating forward selection (SFFS) [51], [52]. The kernel of SFFS is to iteratively select features to maximize the objective function and to remove the unnecessary contents to avoid the local maxima. By the acceptance and rejection process, we obtained the optimal connection features. In this paper, we applied the difference of connection strengths as the objective function and the 12 most discriminative connection features of each section were extracted.

After feature extraction, features among two mental states were classified by some main stream classification, including BP_Adaboost, random forest (RF), relevance vector machine (RVM), and support vector machine (SVM), with the 5fold cross-validation [53]. The radial basis function (RBF) is adopted as the kernel function of SVM (LIBSVM toolbox [54]). The learning method is sequential minimal optimization (SMO). The best $c$ and $g$ were estimated by crossvalidation in the parameter space of $2^{10}, 2^{9}, \ldots, 2^{-9}, 2^{-10}$.

\section{G. Statistical Analysis}

We applied the one-way repeated measures ANOVA for reaction time over the whole simulated driving task to define the alert and fatigue states of EEG data. Then, One-way analysis of variance (ANOVA) was utilized to assess whether or not the changes of the graph theoretical properties were significant from the alert state to fatigue state. It also was used to measure difference of average connection strength between different brain regions and the classification accuracies between different frequency bands. A value of $p<0.05$ was applied to establish the statistical significance. All statistical analyses were performed in the SPSS at the version of 23.0 (IBM, Armonk, New York).

\section{EXPERIMENTAL RESULTS}

\section{A. Behavioral Performance Alteration in Driving Fatigue De- tection}

During the simulated driving task, the behavioral performance reflected the subjects' control ability with time-ontask. The reaction time is a important indicator to distinguish alert and fatigue states. It was recorded over time within a 5-min bin (Fig.2). By separate one-way repeated-measure ANOVA, the significant increment of reaction time $\left(\mathrm{F}_{17,702}=\right.$ 2.740, $p<0.01$ ) were discovered, which indicated the brain control ability declined with the simulated driving task carried out. The human brain achieved the alteration from alert to fatigue state with the time-on-task. Especially, we validated the difference between the first and last 5-min bin, the statistical result suggested the significant different between them $\left(\mathrm{F}_{1,78}\right.$ $=14.392, p<0.01)$. Considering the increasing trend of the reaction time, the brain control ability decreased continuously, therefore, the first and last 5-min with the maximum interval

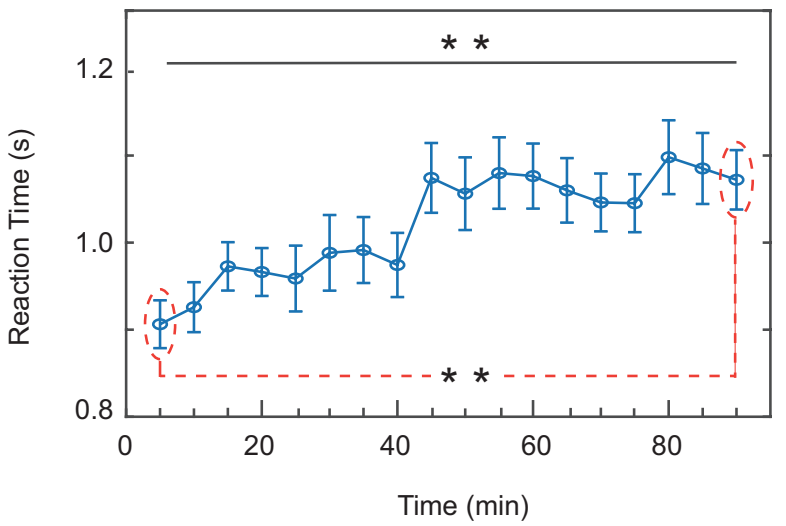

Fig. 2. The reaction time in 5-min bin for the simulated driving task. The curve showed significant increasing trend with the time-on-task. Especially, the significant difference between first and last 5-min were found (dotted line). The error bar is the standard error of the mean across the 40 sections. ** represents $p<0.01$.

and significant difference were defined as the alert and fatigue states in the simulated driving task.

\section{B. Fatigue Effect on Graph Theoretical Properties}

The spatial topology of functional connectivity was measured by graph theory. Table I shows the results of statistical analysis of graph theoretical properties. The great differences of $C^{w}, L^{w}, E_{\text {global }}^{w}$, and $E_{\text {local }}^{w}$ between alert state and fatigue state were discovered $(p<0.05)$ in the considered frequency bands. Particularly, the $L^{w}\left(\theta: \mathrm{F}_{1,78}=5.621, p=0.020 ; \alpha\right.$ : $\left.\mathrm{F}_{1,78}=6.734, p=0.011 ; \beta: \mathrm{F}_{1,78}=9.532, p=0.003\right), E_{\text {global }}^{w}$ $\left(\theta: \mathrm{F}_{1,78}=5.776, p=0.019 ; \alpha: \mathrm{F}_{1,78}=5.723, p=0.019 ;\right.$ $\left.\beta: \mathrm{F}_{1,78}=4.449, p=0.038\right)$ and $E_{\text {local }}^{w}\left(\theta: \mathrm{F}_{1,78}=5.499\right.$, $p=0.022 ; \alpha: \mathrm{F}_{1,78}=6.749, p=0.011 ; \beta: \mathrm{F}_{1,78}=6.868$, $p=0.011$ ) were significantly changed in all these frequency bands. Therefore, the topological structure of brain network changed from alert state and fatigue state, indicating that graph theoretical properties can be used to detect driving fatigue. In terms of the $S W$, there were no significant difference between the alert and fatigue states in total bands, and thus the $S W$ was not included for further driving fatigue detection.

\section{Fatigue Effect on Critical Connections}

The subject-independent critical connections were extracted by the feature selection strategy SFFS. The group averaged critical connections were summarized in Fig.3. The alterations of the connections from alert to fatigue state were estimated. The top $10 \%$ connection strengths of the increased and decreased connections were preserved, respectively. Electrodes followed the disposition of the international 10-20 system. By visual inspection, the top $10 \%$ of the increased connections were related with frontal pole and frontal region: the connections related with these region made up $\theta: 89.29 \% ; \alpha: 78.57 \%$; $\beta: 85.71 \%$ of the total increased connections. Especially, the averaged connection strengths between the nodes in the frontal pole and those in the occipital and parietal regions $(\theta: 0.726$ $\pm 0.106 ; \alpha: 0.880 \pm 0.131 ; \beta: 0.964 \pm 0.163$, mean \pm sem $)$ were generally higher $\left(\mathrm{F}_{1,4}=29.992, p=0.005\right)$ than them in 
Critical Connections
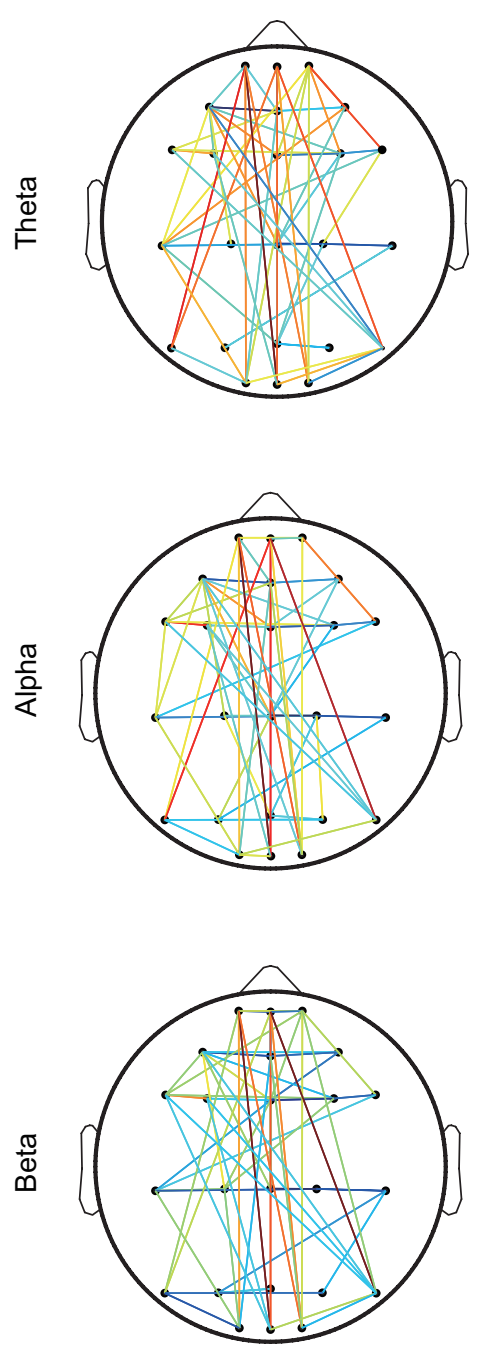

Connection Strength

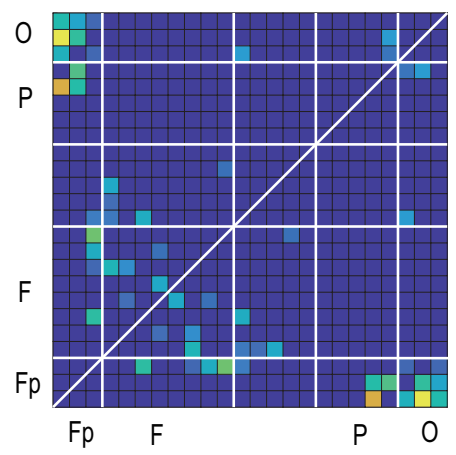

0
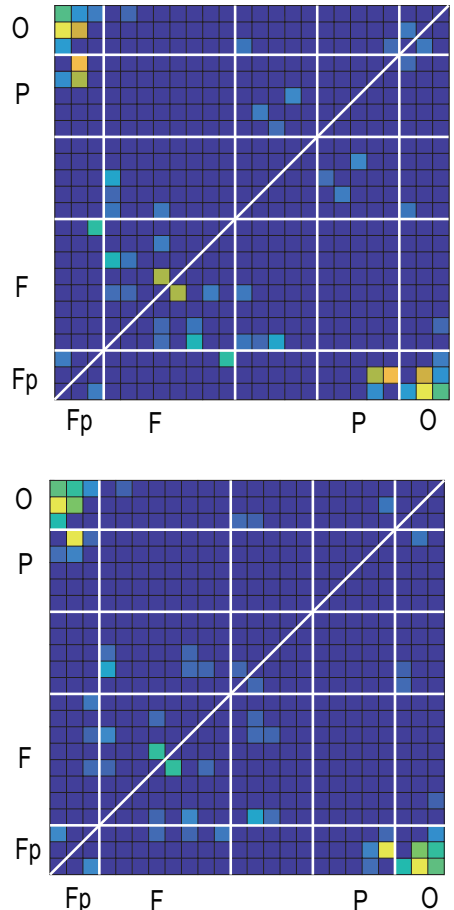

Absolute Value of Alteration

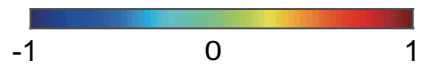

Decreased Connections
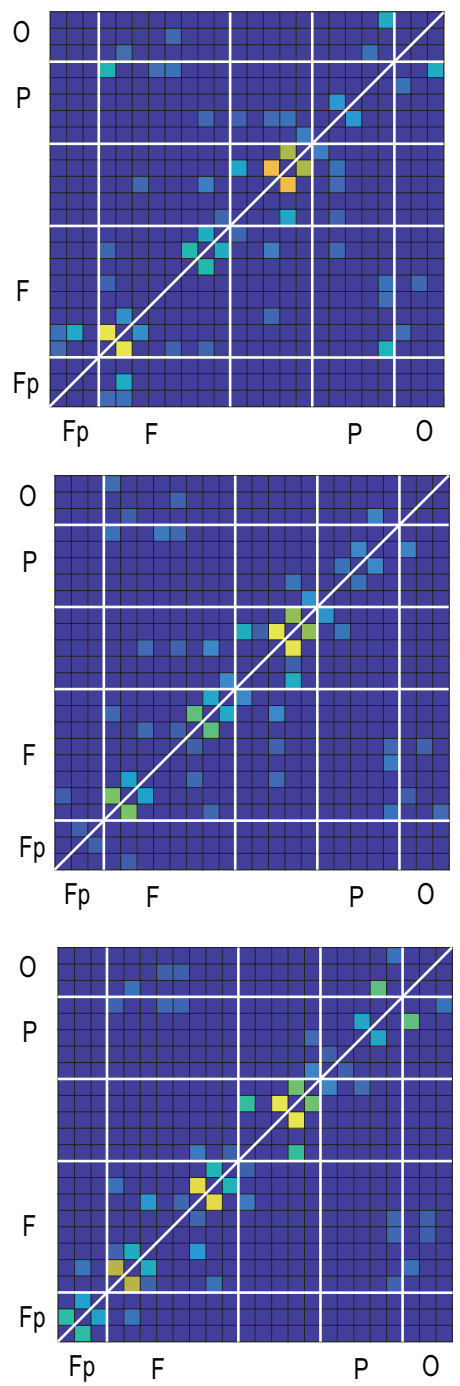

Fig. 3. The group averaged critical connectivity features for driving fatigue detection. The first column is the brain map of the group averaged critical connections. Color bar represents the connection strength and ranged from -1 to 1 . The positive number of the connection strength means it increased from alert to fatigue state and the negative means the inverse result. For better illustration purpose, only the top $10 \%$ connection strengths of the increased and decreased connection are preserved, respectively. The second and third column present the increased and decreased connections of the first column, respectively. Color bar represents the absolute value of the connection strength alterations of increased and decreased connections. Electrodes follow the disposition of the international 10-20 system.

other regions $(\theta: 0.493 \pm 0.041 ; \alpha: 0.464 \pm 0.047 ; \beta: 0.447 \pm$ 0.042 , mean \pm sem), and the percentages of the connections were $35.71 \%$ in $\theta, 32.14 \%$ in $\alpha$ and $35.71 \%$ in $\beta$ of the total increased connections. Furthermore, the connections intrafrontal regions made up $17.86 \%$ in $\theta, 21.43 \%$ in $\alpha$ and $14.29 \%$ in $\beta$ of the total increased connections. On the other hand, the decreased connections mostly scattered in different regions. Interestingly, the intra-regional connections, which resided in frontal pole, frontal, parietal, and occipital regions, made up $25 \%$ in $\theta, 25.71 \%$ in $\alpha$ and $42.86 \%$ in $\beta$ of the total decreased connections. Such results suggested the fatigue-related nodes interactions show the essential local segregation and global integration effect. The frontal region exhibited significant driving fatigue disruptions in all frequency bands. The nodes in the frontal region were more active when driving fatigue occurred.

\section{Classification}

The driving fatigue detection was performed based on the graph theoretical properties and critical connections, respectively. As shown in Fig.4, the SVM classifier showed the best performances in both feature types. Particularly, the mean accuracies of critical connections in three frequency bands $(\theta: 92.33 \% \pm 0.83 \% ; \alpha: 94.09 \% \pm 0.82 \% ; \beta: 96.76 \% \pm$ 
$0.55 \%$, mean \pm sem $)$ are generally higher $\left(\mathrm{F}_{1,4}=73.56\right.$, $p=0.001)$ than graph theoretical properties $(\theta: 75.54 \% \pm$ $1.26 \% ; \alpha: 79.61 \% \pm 1.31 \% ; \beta: 79.68 \% \pm 1.10 \%$, mean \pm sem). Therefore, critical connections are more discriminative for driving fatigue detection. The accuracies in three frequency bands were significant different between each others $\left(\mathrm{F}_{2,117}\right.$ $=8.956, p<0.001)$, which indicated the $\beta$ band was most suitable for driving fatigue detection. Moreover, the higher standard deviation of classification strategy based on graph theoretical properties indicates the larger between-subject differences in the reorganization of the brain functional network at fatigue state.

\section{Discussion}

In our study, we investigated fatigue-related spatial reorganization of the brain network by computing the functional connectivity between alert and fatigue states, aiming to find an efficient approach to achieve the excellent driving fatigue detection. Primarily, we quantitatively measured the topological alteration of the functional connectivity by graph theoretical properties, and these attributes are proved to alter significantly with the progression of driving fatigue. Furthermore, we extracted the most discriminative connections between different mental states. For the two types of features, the classification results suggested that the critical connections were better than its counterpart in driving fatigue detection. According such results, we further analyzed the spatial distribution of critical connections and found that the frontal brain region, especially frontal pole region, was sensitive to driving fatigue based on the observation of the increased and decreased connections. Particularly, these connections showed significant local segregation and global integration effect. More detailed discussions were drawn in the below.

(a)

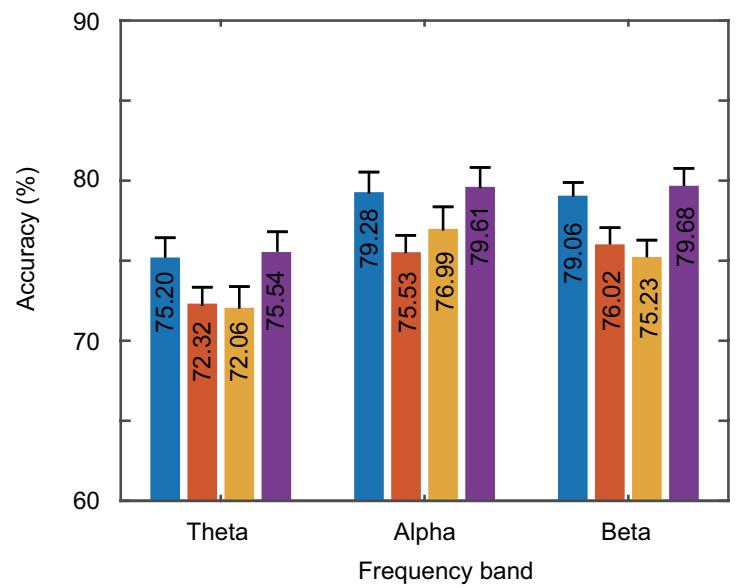

BP Adaboost

\section{A. Brain States Alteration in Graph Theoretical Properties}

Previous studies have investigated the various mental task in the single frequency bands [38], [39], and the essential properties in different bands still lack of research. In this paper, the graph theoretical properties were estimated in three frequency bands to measure the organization of brain functional connectivity. The statistical analysis showed the significant differences $(p<0.05)$ in these properties between alert and fatigue states. In the review of Qi et al. [40], the graph theoretical analysis was employable for mental fatigue detection, such as visual vigilance tasks and sleep deprivation tasks. A study by Vecchio et al. [55] investigated the small world architecture of the brain networks between pre- and post-sleep onset epochs, and their study revealed the sleep onset was related to a less ordered brain network (higher value of small worldness) in the $\sigma$ band as well as a more ordered brain network (lower value of small worldness) in $\delta$ and $\theta$ bands. Therefore, the graph theoretical analysis is applicable in multi-band driving fatigue analysis. The lower global efficiency and longer characteristic path length revealed the brain information processing ability less efficiently. However, the increased clustering coefficient and local efficiency suggested the local neural population were enhanced to resist functional decline. Previous study reported the significant increase in both clustering coefficient and characteristic path length in the lower $\alpha$ band (8 - 10 $\mathrm{Hz}$ ) [38]. This finding is in line with the result in this study. The study in this paper extended the graph theoretical analysis from single band to multi-bands. The singificant alteration of graph theoretical properties in three frequency bands implied the reorganization of the brain functional connectivity. Our results manifested the graph theoretical properties applicable in simulated driving task.

(b)

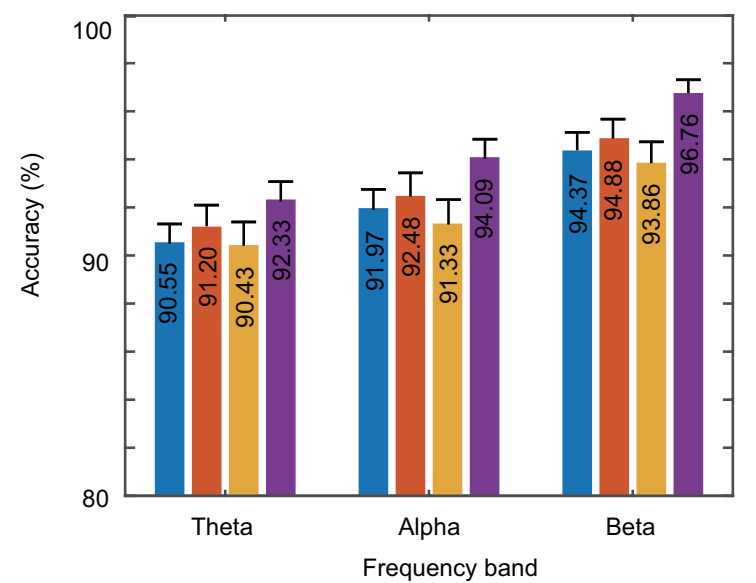

RVM

Fig. 4. The classification accuracies of (a) graph theoretical properties and (b) critical connections from different classification strategies. The accuracies of critical connections are general higher than graph theoretical properties in three frequency bands. Meanwhile, SVM shows the best performance with highest classification accuracies in critical connections compared with other classifiers. For the performance of critical connections, there is no significant differences between different frequency bands. The error bar is the standard error of the mean across the 40 sections. BP_Adaboost: BP_Adaboost classifier; RF: Random Forest classifier; RVM: relevance Vector Machine; SVM: Support Vector Machine. 


\section{B. Topological Alterations of Functional Connectivity Net- works}

Considering the significant differences in graph theoretical properties, which indicated the alteration of brain network, we extracted the most discriminative connections to classify different mental states. Few studies focus on the specific spatial distribution of brain network for mental states recognition. The classification results showed that the critical connection had the dominant performance than the graph theoretical properties. Typically, the $\beta$ band showed the best performance in driving fatigue detection. Such results demonstrated the critical connection could properly reflect the reorganization of brain network between alert and fatigue states, and were sensitive biomarkers for driving fatigue detection.

While drivers feel fatigue, the brain network topology changed for more efficient structure to maintain the brain function [56]. We observed the frontal pole and frontal regions related functional connectivity increased when driver's mental states altered. A more concentrated distribution of the increased connections would lead to the more efficient local information processing ability to resist the brain global function decline. Ishii et al. [57] revealed that the prefrontal cortex and frontal pole play an essential role in the rest decision at mental fatigue state. Inter-regional connections were essential in the attention task to achieve the cooperation between fatigue-related regions, especially the fronto-parietal synchronization [58]. We found the nodes in the frontal pole region are more active to establish connections with other nodes over the whole brain. Previous study indicated the information processing in the brain involved multi-brain region collaboration and neuronal population coordination [59]. Under different mental sates such multi-brain region interaction would be changed to maintain the efficient brain function. In terms of the mental fatigue, studies of neuroscience have unmasked the frontal region is responsible for the sustained attention task [60]. The brain of subjects intended to establish an efficient structure to resist the decline of mental state. For the simulated driving task, the frontal region was still the essential part during the alert and fatigue states. The experimental results suggested, while drivers feel fatigue, some intrafrontal connections increased to improve the regional function. Furthermore, the occipital lobe is related with visual task. Studies have suggested the visual search related mental fatigue detection applied the power spectrum in occipital regions [61]. While driving a car, the driver is needed to achieve sustained visual task to observe the driving condition. This may lead the increased connections between the frontal pole and the occipital regions.

In terms of the decreased connections, we found the distribution of connections are separate that, for the most brain regions, not only the intra-region connections but also the inter-region connections decreased. Even some intra-frontal connections increased, some connections were weakened. This may reveal that even the brain tries to maintain its function, the driving fatigue leads to the decline of the brain information processing ability. The connection decreased in the global scale, this may indicate the decline of the brain function at most brain regions.

Even the networks of graph theoretical properties and critical connections were extracted by different methods respectively (the sparsity strategy and the SFFS), the similar features were discovered. The increased connections are clustered between the driving fatigue-related regions, like frontal pole, frontal, and parietal region. Thus, the function of these brain driving fatigue-related regions converted to better information processing ability. Such performances are in line with the alterations of the graph theoretical properties that higher clustering coefficient and local efficiency in fatigue state. For the decreased connections, most of them are scattered at the whole brain regions. Such results lead to the decrement of the global information processing ability with lower global efficiency and higher characteristic path length at fatigue state. When driver feel drowsy, the brain information spreading ability is declined with the lower communication efficiency, as a result the intra- and inter-regions connections were generally weakened. However, the brain try to maintain the driving task. It strengthens the connections of special brain regions with the function of cognition, visual, and body control with the efficient information communication ability between these regions. The brain function showed strong global integration and local segregation, the critical connections showed that brain functional structure is a network with small-world property [62]-[65]. Therefore, investigating the critical statistical correlation based on the interaction between brain regions can provide critical insights into the neural activities for neuroimaging research.

\section{Driving Fatigue Recognition}

In the functional connectivity based driving fatigue detection field, the previous studies mostly focused on the alterations of the connections or graph theoretical properties and their statistical differences [67], [70], as shown in Table II. For the practical application, Liu [66] and his colleagues recorded the EEG data from the actual driving experiment and established effective network to distinguish mental states. Wang et al. [68] developed the danger point prediction system to monitor driver's mental state in the real driving condition. However, these studies revealed the essential mechanisms of the brain information transmission, but not the driving fatigue classification strategy. Lacking the classification performance, these systems cannot been known as the applicable driving fatigue detection system to judge the drivers mental state. In the study of Chen et al. [69], they only measured the $C$ and $L$ as the graph theoretical properties to achieve such system. These studies analyzed the limited mechanism of driving fatigue detection in a single aspect, such as critical connections or graph theoretical properties. Even in an aspect, like graph theoretical properties, the studies based on small number of parameters $\left(C, L\right.$ or $\left.C, E_{\text {global }}\right)$ cannot reflect the whole brain activity to measure the local segregation and global integration of brain function. Also, few of them achieved the real driving fatigue detection: the driving fatigue classification with high accuracy. Our previous study [39] using the critical connections achieved the driving fatigue classification in single 
TABLE II

SUMMARY OF FUNCTIONAL CONNECTIVITY-RELATED DRIVING FATIGUE DETECTION METHODS.

\begin{tabular}{|c|c|c|c|c|c|c|}
\hline Ref. & $\begin{array}{c}\text { Experiment } \\
\text { paradigm }\end{array}$ & Frequency bands & Channel number & Edge definition & Network features & $\begin{array}{c}\text { Classification } \\
\text { accuracy }\end{array}$ \\
\hline [66] & $\begin{array}{l}\text { Actual driving } \\
\text { experiment }\end{array}$ & $\delta$ & 16 channels & GC & Str & N/A \\
\hline [67] & $\begin{array}{l}\text { Actual driving } \\
\text { experiment }\end{array}$ & $\begin{array}{c}\theta, \beta, \text { and high slow } \\
\gamma\end{array}$ & 14 channels & Pearson correlation & $C$ and $E_{\text {global }}^{w}$ & N/A \\
\hline [68] & $\begin{array}{c}\text { Actual driving } \\
\text { experiment }\end{array}$ & $\alpha$ & 19 channels & FSL & Str & N/A \\
\hline [69] & $\begin{array}{l}\text { Actual driving } \\
\text { experiment }\end{array}$ & $\delta, \theta, \alpha$, and $\beta$ & 14 channels & PLI & $C$ and $L$ & $94.4 \%$ \\
\hline [70] & $\begin{array}{l}\text { Simulated driving } \\
\text { experiment }\end{array}$ & $\delta, \theta, \alpha$, and $\beta$ & 30 channels & PLI & Gt & N/A \\
\hline [71] & $\begin{array}{c}\text { Simulated driving } \\
\text { experiment }\end{array}$ & $\delta, \theta, \alpha, \beta$, and $\gamma$ & 64 and 16 channels & MPC & Str & N/A \\
\hline [39] & $\begin{array}{l}\text { Simulated driving } \\
\text { experiment }\end{array}$ & $\theta$ & 64 channels & GPDC & Cri & $92 \%$ \\
\hline this paper & $\begin{array}{c}\text { Simulated driving } \\
\text { experiment }\end{array}$ & $\theta, \alpha$, and $\beta$ & 24 channels & PLI & Gt or Cri & $96.76 \%$ \\
\hline
\end{tabular}

GC: Granger causality; FSL: fuzzy synchronization likelihood; MPC: mean phase coherence; GPDC: general partial directed coherence; Str: connection strength; Cri: critical connections; Gt: graph theoretical properties.

frequency band, the multi-bands performances are lacking indepth investigation. Not only the neural mechanism but also the classification of both two graph and connection features in different frequency bands need further research. Therefore, in this paper, we creatively measured two aspects of the brain activity and compared their performances in driving fatigue detection in different frequency bands.

We employed four types of classifiers (BP Adaboost, random forest $(\mathrm{RF})$, relevance vector machine (RVM), and support vector machine (SVM)) to assess the performances of functional connectivity networks. For the graph theoretical properties, the SVM achieved the best classification accuracies in three frequency bands. At the same time, the BP_Adaboost obtained the secondary performances. The RVM showed the worst results in $\theta$ and $\beta$ bands, and the FR showed the worst result in the $\alpha$ band.

On the other hand, the driving fatigue detection performances of critical connections in four types of classifiers were generally higher than the counterparts of graph theoretical properties. The critical connections were most suitable with SVM, which classification accuracies were the highest in all bands. Furthermore, RF showed the secondary performances across all frequency bands. For BP_Adaboost, it obtained the third performances as well as the RVM obtained the worst results.

\section{Experimental Limitation}

One limitation of the current study is that fusion of the graph theoretical properties and critical connections features are not achieved for driving fatigue detection. The statistical analysis suggested the graph theoretical properties significant changed from alert to fatigue state that the fusion of both features may provide better performance. The future work may investigate the fusion features in the driving fatigue detection. Furthermore, the critical connections were independent across each section, and such a method was not suitable for the actual driving condition. The non-independent features across all sections are lacking of investigating. The future work should develop the cross subject features for the actual driving fatigue detection.

\section{CONClusion}

This study investigated the driving fatigue detection based on the reorganization of functional connectivity between alert and fatigue states. The graph theoretical properties and critical connections were extracted from functional networks. The critical connections were suggested as the dominant features for the driving fatigue detection. We further analyzed the spatial distribution of critical connections, which manifested the frontal pole and frontal regions were more active while driving fatigue occurred. Especially, the increment and decrement of the functional connectivity showed significant global integration and local segregation while driving fatigue occurred. Our findings suggest the critical connections is a feasible biomarker for driving fatigue detection, and provide a new approach for efficient driving fatigue detection.

\section{REFERENCES}

[1] G. Borghini, L. Astolfi, G. Vecchiato, D. Mattia, and F. Babiloni, "Measuring neurophysiological signals in aircraft pilots and car drivers for the assessment of mental workload, fatigue and drowsiness," Neuroscience \& Biobehavioral Reviews, vol. 44, pp. 58-75, 2014.

[2] R. A. Grier, J. S. Warm, W. N. Dember, G. Matthews, T. L. Galinsky, J. L. Szalma, and R. Parasuraman, "The vigilance decrement reflects limitations in effortful attention, not mindlessness," Human Factors, vol. 45 , no. 3, pp. 349-359, 2003.

[3] P. S. Rau, "Drowsy driver detection and warning system for commercial vehicle drivers: field operational test design, data analyses, and progress," National Highway Traffic Safety Administration, pp. 05-0192, 2005.

[4] S. K. Lal and A. Craig, "A critical review of the psychophysiology of driver fatigue," Biological psychology, vol. 55, no. 3, pp. 173-194, 2001.

[5] G. Matthews and P. A. Desmond, "Task-induced fatigue states and simulated driving performance," The Quarterly Journal of Experimental Psychology Section A, vol. 55, no. 2, pp. 659-686, 2002. 
[6] C.-S. Huang, N. R. Pal, C.-H. Chuang, and C.-T. Lin, "Identifying changes in eeg information transfer during drowsy driving by transfer entropy," Frontiers in Human Neuroscience, vol. 9, p. 570, 2015.

[7] S. M. Marcora, W. Staiano, and V. Manning, "Mental fatigue impairs physical performance in humans," Journal of Applied Physiology, vol. 106, no. 3, pp. 857-864, 2009.

[8] W. Rong-Ben, G. Ke-You, S. Shu-Ming, and C. Jiang-Wei, "A monitoring method of driver fatigue behavior based on machine vision," in IEEE IV2003 Intelligent Vehicles Symposium. Proceedings. IEEE, 2003, pp. $110-113$.

[9] W.-B. Horng, C.-Y. Chen, Y. Chang, and C.-H. Fan, "Driver fatigue detection based on eye tracking and dynamk, template matching," in 2004 IEEE International Conference on Networking, Sensing and Control, vol. 1. IEEE, 2004, pp. 7-12.

[10] E. Vural, M. Cetin, A. Ercil, G. Littlewort, M. Bartlett, and J. Movellan, "Drowsy driver detection through facial movement analysis," in International Workshop on Human-Computer Interaction. S Springer, 2007, pp. 6-18.

[11] Q. Wang, J. Yang, M. Ren, and Y. Zheng, "Driver fatigue detection: a survey," in Intelligent Control and Automation, 2006. WCICA 2006. The Sixth World Congress on, vol. 2. IEEE, 2006, pp. 8587-8591.

[12] M. Simon, E. A. Schmidt, W. E. Kincses, M. Fritzsche et al., "Eeg alpha spindle measures as indicators of driver fatigue under real traffic conditions," Clinical Neurophysiology, vol. 122, no. 6, pp. 1168-1178, 2011.

[13] C. Zhao, M. Zhao, J. Liu, and C. Zheng, "Electroencephalogram and electrocardiograph assessment of mental fatigue in a driving simulator," Accident Analysis \& Prevention, vol. 45, pp. 83-90, 2012.

[14] R. N. Khushaba, S. Kodagoda, S. Lal, and G. Dissanayake, "Driver drowsiness classification using fuzzy wavelet-packet-based featureextraction algorithm," IEEE Transactions on Biomedical Engineering, vol. 58, no. 1, pp. 121-131, 2011.

[15] C.-H. Chuang, C.-S. Huang, L.-W. Ko, and C.-T. Lin, "An eeg-based perceptual function integration network for application to drowsy driving," Knowledge-Based Systems, vol. 80, pp. 143-152, 2015.

[16] L. H. Chew, J. Teo, and J. Mountstephens, "Aesthetic preference recognition of 3d shapes using eeg," Cognitive Neurodynamics, vol. 10, no. 2 , pp. 165-173, 2016.

[17] P. Atchley, M. Chan, and S. Gregersen, "A strategically timed verbal task improves performance and neurophysiological alertness during fatiguing drives," Human Factors, vol. 56, no. 3, pp. 453-462, 2014.

[18] J. Harvy, E. Sigalas, N. V. Thakor, A. Bezerianos, and J. Li, "Performance improvement of driving fatigue identification based on power spectra and connectivity using feature level and decision level fusions."

[19] J. He, G. Zhou, H. Wang, E. Sigalas, N. Thakor, A. Bezerianos, and J. Li, "Boosting transfer learning improves performance of driving drowsiness classification using eeg," in 2018 International Workshop on Pattern Recognition in Neuroimaging (PRNI). IEEE, 2018, pp. 1-4.

[20] R. Bose, H. Wang, A. Dragomir, N. V. Thakor, A. Bezerianos, and $\mathrm{J}$. Li, "Regression based continuous driving fatigue estimation: Towards practical implementation," IEEE Transactions on Cognitive and Developmental Systems, 2019.

[21] N. Gurudath and H. B. Riley, "Drowsy driving detection by eeg analysis using wavelet transform and k-means clustering," Procedia Computer Science, vol. 34, pp. 400-409, 2014.

[22] Y. Xiong, J. Gao, Y. Yang, X. Yu, and W. Huang, "Classifying driving fatigue based on combined entropy measure using eeg signals," International Journal of Control and Automation, vol. 9, no. 3, pp. 329-338, 2016.

[23] F. Wang, H. Wang, and R. Fu, "Real-time ecg-based detection of fatigue driving using sample entropy," Entropy, vol. 20, no. 3, p. 196, 2018.

[24] H. Wang, A. Dragomir, N. I. Abbasi, J. Li, N. V. Thakor, and A. Bezerianos, "A novel real-time driving fatigue detection system based on wireless dry eeg," Cognitive Neurodynamics, vol. 2, pp. 365-376, 2018.

[25] J. Harvy, N. V. Thakor, A. Bezerianos, and J. Li, "Between-frequency topographical and dynamic high-order functional connectivity for driving drowsiness assessment," IEEE Transactions on Neural Systems and Rehabilitation Engineering, vol. 27, no. 3, pp. 358-367, 2019.

[26] G. N. Dimitrakopoulos, I. Kakkos, A. G. Vrahatis, K. N. Sgarbas, J. Li, Y. Sun, and A. Bezerianos, "Driving mental fatigue classification based on brain functional connectivity," in International Conference on Engineering Applications of Neural Networks, 2017, pp. 465-474.

[27] O. Sporns, "The human connectome: a complex network," Annals of the New York Academy of Sciences, vol. 1224, no. 1, pp. 109-125, 2011.

[28] M. A. Boksem, T. F. Meijman, and M. M. Lorist, "Effects of mental fatigue on attention: an erp study," Cognitive Brain Research, vol. 25, no. 1, pp. 107-116, 2005.
[29] H.-J. Park and K. Friston, "Structural and functional brain networks: from connections to cognition," Science, vol. 342, no. 6158, p. 1238411, 2013.

[30] S. Micheloyannis, E. Pachou, C. J. Stam et al., "Using graph theoretical analysis of multi channel eeg to evaluate the neural efficiency hypothesis," Neuroscience Letters, vol. 402, no. 3, pp. 273-277, 2006.

[31] Y. Sun, J. Lim, J. Meng, K. Kwok, N. Thakor, and A. Bezerianos, "Discriminative analysis of brain functional connectivity patterns for mental fatigue classification," Annals of Biomedical Engineering, vol. 42, no. 10, pp. 2084-2094, 2014

[32] J. Li, J. Lim, Y. Chen, K. Wong, N. Thakor, A. Bezerianos, and Y. Sun, "Mid-task break improves global integration of functional connectivity in lower alpha band," Frontiers in Human Neuroscience, vol. 10, p. 304, 2016.

[33] T. P. Breckel, C. M. Thiel, E. T. Bullmore, A. Zalesky, A. X. Patel, and C. Giessing, "Long-term effects of attentional performance on functional brain network topology," PloS One, vol. 8, no. 9, p. e74125, 2013.

[34] Z. Dai, J. De Souza, J. Lim, P. M. Ho, Y. Chen, J. Li, N. Thakor, A. Bezerianos, and Y. Sun, "Eeg cortical connectivity analysis of working memory reveals topological reorganization in theta and alpha bands," Frontiers in Human Neuroscience, vol. 11, p. 237, 2017.

[35] J. Lim, W.-c. Wu, J. Wang, J. A. Detre, D. F. Dinges, and H. Rao, "Imaging brain fatigue from sustained mental workload: an asl perfusion study of the time-on-task effect," Neuroimage, vol. 49, no. 4, pp. 3426$3435,2010$.

[36] J.-P. Liu, C. Zhang, and C.-X. Zheng, "Estimation of the cortical functional connectivity by directed transfer function during mental fatigue," Applied Ergonomics, vol. 42, no. 1, pp. 114-121, 2010.

[37] K. C. Bettencourt and Y. Xu, "Decoding the content of visual shortterm memory under distraction in occipital and parietal areas," Nature Neuroscience, vol. 19, no. 1, p. 150, 2016.

[38] Y. Sun, J. Lim, K. Kwok, and A. Bezerianos, "Functional cortical connectivity analysis of mental fatigue unmasks hemispheric asymmetry and changes in small-world networks," Brain and Cognition, vol. 85, pp. 220-230, 2014

[39] G. N. Dimitrakopoulos, I. Kakkos, Z. Dai, H. Wang, K. Sgarbas, N. Thakor, A. Bezerianos, and Y. Sun, "Functional connectivity analysis of mental fatigue reveals different network topological alterations between driving and vigilance tasks," IEEE Transactions on Neural Systems and Rehabilitation Engineering, vol. 26, no. 4, pp. 740-749, 2018.

[40] P. Qi, H. Ru, L. Gao, X. Zhang, T. Zhou, Y. Tian, N. Thakor, A. Bezerianos, J. Li, and Y. Sun, "Neural mechanisms of mental fatigue revisited: New insights from the brain connectome," Engineering, 2019.

[41] U. Herwig, P. Satrapi, and C. Schönfeldt-Lecuona, "Using the international 10-20 eeg system for positioning of transcranial magnetic stimulation," Brain Topography, vol. 16, no. 2, pp. 95-99, 2003.

[42] C. J. James and C. W. Hesse, "Independent component analysis for biomedical signals," Physiological Measurement, vol. 26, no. 1, p. R15, 2004.

[43] R. R. Coifman, Y. Meyer, S. Quake, and M. V. Wickerhauser, "Signal processing and compression with wavelet packets," in Wavelets and their Applications. Springer, 1994, pp. 363-379.

[44] A. Delorme and S. Makeig, "Eeglab: an open source toolbox for analysis of single-trial eeg dynamics including independent component analysis," Journal of Neuroscience Methods, vol. 134, no. 1, pp. 9-21, 2004.

[45] M. Hassan and F. Wendling, "Aiming for high resolution of brain networks in time and space electroencephalography source connectivity," IEEE Signal Processing Magazine, vol. 35, no. 3, pp. 81-96, 2017.

[46] S. Aydore, D. Pantazis, and R. M. Leahy, "A note on the phase locking value and its properties," Neuroimage, vol. 74, pp. 231-244, 2013.

[47] M. Rubinov and O. Sporns, "Complex network measures of brain connectivity: uses and interpretations," Neuroimage, vol. 52, no. 3, pp. $1059-1069,2010$.

[48] J. Saramäki, M. Kivelä, J.-P. Onnela, K. Kaski, and J. Kertesz, "Generalizations of the clustering coefficient to weighted complex networks," Physical Review E, vol. 75, no. 2, p. 027105, 2007.

[49] S. Maslov and K. Sneppen, "Specificity and stability in topology of protein networks," Science, vol. 296, no. 5569, pp. 910-913, 2002.

[50] M. D. Humphries, K. Gurney, and T. J. Prescott, "The brainstem reticular formation is a small-world, not scale-free, network," Proceedings of the Royal Society B: Biological Sciences, vol. 273, no. 1585, pp. 503-511, 2005.

[51] P. Pudil, F. J. Ferri, J. Novovicova, and J. Kittler, "Floating search methods for feature selection with nonmonotonic criterion functions," in 12th IAPR International Conference on Pattern Recognition, vol. 2. IEEE, 1994, pp. 279-283. 
[52] I. Kakkos, E. M. Ventouras, P. A. Asvestas et al., "A conditionindependent framework for the classification of error-related brain activity," Medical \& Biological Engineering \& Computing, pp. 1-15, 2020.

[53] Y. Zhang, B. Liu, X. Ji, and D. Huang, "Classification of eeg signals based on autoregressive model and wavelet packet decomposition," Neural Processing Letters, vol. 45, no. 2, pp. 365-378, 2017.

[54] C.-C. Chang and C.-J. Lin, "Libsvm: a library for support vector machines," ACM Transactions on Intelligent Systems and Technology (TIST), vol. 2, no. 3, p. 27, 2011.

[55] F. Vecchio, F. Miraglia, M. Gorgoni, M. Ferrara, F. Iberite, P. Bramanti, L. De Gennaro, and P. M. Rossini, "Cortical connectivity modulation during sleep onset: a study via graph theory on eeg data," Human Brain Mapping, vol. 38, no. 11, pp. 5456-5464, 2017.

[56] L. Xu, B. Wang, G. Xu, W. Wang, Z. Liu, and Z. Li, "Functional connectivity analysis using fnirs in healthy subjects during prolonged simulated driving," Neuroscience Letters, vol. 640, pp. 21-28, 2017.

[57] A. Ishii, M. Tanaka, and Y. Watanabe, "The neural mechanisms underlying the decision to rest in the presence of fatigue: a magnetoencephalography study," PloS One, vol. 9, no. 10, 2014.

[58] M. S. Clayton, N. Yeung, and R. C. Kadosh, "The roles of cortical oscillations in sustained attention," Trends in Cognitive Sciences, vol. 19, no. 4, pp. 188-195, 2015.

[59] B. Horwitz, "The elusive concept of brain connectivity," Neuroimage, vol. 19, no. 2, pp. 466-470, 2003.

[60] N. U. Dosenbach, D. A. Fair, and Miezin, "Distinct brain networks for adaptive and stable task control in humans," Proceedings of the National Academy of Sciences, vol. 104, no. 26, pp. 11 073-11 078, 2007.

[61] X. Fan, Q. Zhou, Z. Liu, and F. Xie, "Electroencephalogram assessment of mental fatigue in visual search," Bio-medical Materials and Engineering, vol. 26, no. s1, pp. S1455-S1463, 2015.

[62] U. Braun, S. F. Muldoon, and D. S. Bassett, "On human brain networks in health and disease," $e L S$, pp. 1-9, 2001.

[63] M. P. van den Heuvel and O. Sporns, "Network hubs in the human brain," Trends in Cognitive Sciences, vol. 17, no. 12, pp. 683-696, 2013.

[64] X. Liu, T. Li, C. Tang, T. Xu, P. Chen, A. Bezerianos, and H. Wang, "Emotion recognition and dynamic functional connectivity analysis based on eeg," IEEE Access, vol. 7, pp. 143 293-143 302, 2019.

[65] D. J. Watts and S. H. Strogatz, "Collective dynamics of smallworldnetworks," Nature, vol. 393, no. 6684, p. 440, 1998.

[66] Z. Liu, M. Zhang, G. Xu, C. Huo, Q. Tan, Z. Li, and Q. Yuan, "Effective connectivity analysis of the brain network in drivers during actual driving using near-infrared spectroscopy," Frontiers in Behavioral Neuroscience, vol. 11, 2017.

[67] F. Wang, X. Zhang, R. Fu, and G. Sun, "Eeg characteristic analysis of coach bus drivers based on brain connectivity as revealed via a graph theoretical network," RSC Advances, vol. 8, no. 52, pp. 29745-29755, 2018.

[68] H. Wang, C. Zhang, T. Shi, F. Wang, and S. Ma, "Real-time eeg-based detection of fatigue driving danger for accident prediction," International Journal of Neural Systems, vol. 25, no. 02, p. 1550002, 2015.

[69] J. Chen, H. Wang, Q. Wang, and C. Hua, "Exploring the fatigue affecting electroencephalography based functional brain networks during real driving in young males," Neuropsychologia, vol. 129, pp. 200-211, 2019.

[70] J. Chen, H. Wang, C. Hua, Q. Wang, and C. Liu, "Graph analysis of functional brain network topology using minimum spanning tree in driver drowsiness," Cognitive Neurodynamics, vol. 12, no. 6, pp. 569$581,2018$.

[71] W. Kong, Z. Zhou, B. Jiang, F. Babiloni, and G. Borghini, "Assessment of driving fatigue based on intra/inter-region phase synchronization," Neurocomputing, vol. 219, pp. 474-482, 2017. 